TRANSACTIONS OF THE

AMERICAN MATHEMATICAL SOCIETY

Volume 357, Number 1, Pages 179-196

S 0002-9947(04)03548-2

Article electronically published on March 23, 2004

\title{
ON A REFINEMENT OF THE GENERALIZED CATALAN NUMBERS FOR WEYL GROUPS
}

\author{
CHRISTOS A. ATHANASIADIS
}

\begin{abstract}
Let $\Phi$ be an irreducible crystallographic root system with Weyl group $W$, coroot lattice $\check{Q}$ and Coxeter number $h$, spanning a Euclidean space $V$, and let $m$ be a positive integer. It is known that the set of regions into which the fundamental chamber of $W$ is dissected by the hyperplanes in $V$ of the form $(\alpha, x)=k$ for $\alpha \in \Phi$ and $k=1,2, \ldots, m$ is equinumerous to the set of orbits of the action of $W$ on the quotient $\check{Q} /(m h+1) \check{Q}$. A bijection between these two sets, as well as a bijection to the set of certain chains of order ideals in the root poset of $\Phi$, are described and are shown to preserve certain natural statistics on these sets. The number of elements of these sets and their corresponding refinements generalize the classical Catalan and Narayana numbers, which occur in the special case $m=1$ and $\Phi=A_{n-1}$.
\end{abstract}

\section{INTRODUCTION AND RESULTS}

Let $V$ be an $\ell$-dimensional Euclidean space, with inner product (, ), and let $\Phi$ be an irreducible crystallographic root system spanning $V$. Let $m$ be a fixed nonnegative integer. We denote by $\mathrm{Cat}_{\Phi}^{m}$ the collection of hyperplanes in $V$ defined by the affine equations $(\alpha, x)=k$ for $\alpha \in \Phi$ and $k=0,1, \ldots, m$. Thus $\operatorname{Cat}_{\Phi}^{m}$ is a deformation of the Coxeter arrangement $\mathcal{A}_{\Phi}$, in the sense of [1, 16, known as an extended Catalan arrangement. It is invariant under the action of the Weyl group $W$ associated to $\Phi$ and reduces to $\mathcal{A}_{\Phi}$ for $m=0$. The Weyl group $W$ acts on the coroot lattice $\check{Q}$ of $\Phi$ and its dilate $(m h+1) \check{Q}$, where $h$ denotes the Coxeter number of $\Phi$. Hence $W$ acts also on the quotient

$$
\check{Q} /(m h+1) \check{Q} .
$$

We denote this quotient by $T^{m}$. For a fixed choice of a positive system $\Phi^{+} \subseteq \Phi$, consider the partial order on $\Phi^{+}$defined by letting $\alpha \leq \beta$ if $\beta-\alpha$ is a nonnegative linear combination of positive roots, known as the root poset of $\Phi$. A (dual) order ideal in $\Phi^{+}$is a subset $I$ of $\Phi^{+}$such that $\alpha \in I$ and $\alpha \leq \beta$ in $\Phi^{+}$imply $\beta \in I$.

Our starting point is the following theorem, on the origins of which we comment later in this section.

Theorem 1.1 (2, 10, 21]). Let $\Phi$ be an irreducible crystallographic root system with Weyl group $W$, and let $m$ be a nonnegative integer.

Received by the editors March 16, 2003 and, in revised form, June 26, 2003.

2000 Mathematics Subject Classification. Primary 20F55; Secondary 05E99, $20 \mathrm{H} 15$.

Key words and phrases. Weyl group, coroot lattice, root order, ideals, hyperplane arrangements, regions, Narayana numbers. 
The number of regions into which the fundamental chamber of $\mathcal{A}_{\Phi}$ is dissected by the hyperplanes of $\mathrm{Cat}_{\Phi}^{m}$ is equal to the number of orbits of the action of $W$ on $T^{m}$ and is given by the product

$$
\prod_{i=1}^{\ell} \frac{e_{i}+m h+1}{e_{i}+1},
$$

where $\ell$ is the rank, $h$ is the Coxeter number and $e_{1}, e_{2}, \ldots, e_{\ell}$ are the exponents of $\Phi$. Moreover, for $m=1$ this number is equal to the number of ideals in the root poset of $\Phi$.

To state our main result we need to introduce some more notation and definitions. For $y \in T^{m}$, consider the stabilizer of $y$ with respect to the $W$-action on $T^{m}$. This is a subgroup of $W$ generated by reflections. We call the minimum number of reflections needed to generate this subgroup its rank and denote it by $r(y)$. We may use the notation $r(x)$ for a $W$-orbit $x$ in $T^{m}$, since stabilizers of elements of $T^{m}$ in the same $W$-orbit are conjugate subgroups of $W$ and hence have the same rank. We denote by $H_{\alpha, k}$ the affine hyperplane in $V$ defined by the equation $(\alpha, x)=k$, and by $A_{\circ}$ the fundamental alcove of the affine Weyl arrangement corresponding to $\Phi$. A wall of a region $R$ of $\mathrm{Cat}_{\Phi}^{m}$ is a hyperplane in $V$ which supports a facet of $R$. We call a decreasing chain $\Phi^{+}=I_{0} \supseteq I_{1} \supseteq I_{2} \supseteq \cdots \supseteq I_{m}$ of ideals in $\Phi^{+}$a co-filtered chain of ideals of length $m$ if

$$
\left(I_{i}+I_{j}\right) \cap \Phi^{+} \subseteq I_{i+j}
$$

holds for all indices $i, j$, where $I_{i}=I_{m}$ for $i>m$, and

$$
\left(J_{i}+J_{j}\right) \cap \Phi^{+} \subseteq J_{i+j}
$$

holds for all indices $i, j \geq 1$ with $i+j \leq m$, where $J_{i}=\Phi^{+} \backslash I_{i}$. A positive root $\alpha$ is indecomposable of rank $m$ with respect to this chain if $\alpha \in I_{m}$ and it is not possible to write $\alpha=\beta+\gamma$ with $\beta \in I_{i}$ and $\gamma \in I_{j}$ for indices $i, j \geq 0$ with $i+j=m$. The following theorem gives a refinement of Theorem 1.1.

Theorem 1.2. Let $\Phi$ be an irreducible crystallographic root system with Weyl group $W$, let $m$ be a positive integer and let $O^{m}(\Phi)$ be the set of orbits of the action of $W$ on $T^{m}$. For any nonnegative integer $i$, the following are equal:

(i) the number of regions $R$ of $\mathrm{Cat}_{\Phi}^{m}$ in the fundamental chamber of $\mathcal{A}_{\Phi}$ such that $i$ walls of $R$ of the form $H_{\alpha, m}$ separate $R$ from $A_{\circ}$,

(ii) the number of orbits $x \in O^{m}(\Phi)$ with $r(x)=i$, and

(iii) the number of co-filtered chains of ideals in the root poset $\Phi^{+}$of length $m$ having $i$ indecomposable elements of rank $m$.

In particular, the number of co-filtered chains of ideals in $\Phi^{+}$of length $m$ is equal to the product (1.1).

In the special case $m=1$ a co-filtered chain of ideals consists of a single ideal $I$ in $\Phi$ and the set of rank one indecomposable elements is the set of minimal elements of $I$, which is an antichain in $\Phi^{+}$. The arrangement $\mathrm{Cat}_{\Phi}^{m}$ in this case consists of the hyperplanes $H_{\alpha}$ and $H_{\alpha, 1}$ for all $\alpha \in \Phi$ and is known as the Catalan arrangement associated to $\Phi$, denoted $\mathrm{Cat}_{\Phi}$. The equality between the quantities in (i) and (iii) of the following corollary of Theorem 1.2 was conjectured by S. Fomin and J.R. Stembridge and independently by F. Chapoton. It was obtained earlier in 
an equivalent form (via Lemma 4.1) by Panyushev [14] and is also implicit in the recent work of Sommers 22] (see Proposition 6.2 there).

Corollary 1.3. Let $\Phi$ be an irreducible crystallographic root system with Weyl group $W$, and let $O(\Phi)$ be the set of orbits of the action of $W$ on $T=\check{Q} /(h+1) \check{Q}$. For any nonnegative integer $i$, the following are equal:

(i) the number of $i$-element antichains in the root poset of $\Phi$,

(ii) the number of regions $R$ of $\mathrm{Cat}_{\Phi}$ in the fundamental chamber of $\mathcal{A}_{\Phi}$ such that $i$ walls of $R$ separate $R$ from $A_{\circ}$, and

(iii) the number of orbits $x \in O(\Phi)$ with $r(x)=i$.

A few comments on Theorem 1.1 are in order. Formula (1.1) was proved for the number of regions of $\mathrm{Cat}_{\Phi}^{m}$ in the fundamental chamber by the author [2, Corollary 1.3] (see also [1, Theorem 4.6]) and for the number of $W$-orbits of $\check{Q} / k \check{Q}$ (with $m h+1$ replaced by $k$ ) by Haiman [10, Theorem 7.4.2] under a hypothesis on $k$ which includes the cases $k=m h+1$. The same formula for the number of such orbits was earlier proved by Djoković [7, page 183] under a more restrictive assumption, namely that $k$ is relatively prime to the order of $W$. The number of ideals in $\Phi^{+}$was computed case by case by Shi 21, and by A. Postnikov (unpublished) in the cases of the classical root systems, and was observed in [17] to fit the uniform formula (1.1) for $m=1$. In his study of sign types for affine Weyl groups [20, 21], Shi also showed that the set of ideals in $\Phi^{+}$is in bijection with the set of positive sign types for $W$ and with the set of regions into which the fundamental chamber is dissected by the hyperplanes of Cat $\Phi$. A bijection between ideals in $\Phi^{+}$and $W$-orbits of $T$ was later described by Cellini and Papi [5] and is based on the construction of a map which assigns an element of the affine Weyl group $W_{a}$ to each ideal in $\Phi^{+}[4]$.

In the special case $m=1$, the expression (1.1) is refered to as the Catalan number associated to $\Phi$ [2 17, since it reduces to the familiar $n$th Catalan number for the root system $A_{n-1}$. It has a variety of interesting combinatorial interpretations apart from those given in Theorem 1.1, for instance as the number of noncrossing partitions associated to $W$ [3, Proposition 5.2.1], [15, Section 4], as the number of vertices of the simple generalized associahedron associated to $\Phi$ [8, Theorem 1.9], and as the number of clusters in the corresponding cluster algebra of finite type 9]. No case-free proofs of any of these facts are known. The numbers which appear in Corollary 1.3 refine this Catalan number and are known as the Narayana numbers [12] in the case of the root system $A_{n-1}$. Therefore they may be refered to as the Narayana numbers associated to $\Phi$. It has been verified case by case that the same numbers appear as the rank numbers of the poset of noncrossing partitions associated to $W$ and as the entries of the $h$-vector of the simplicial generalized associahedron associated to $\Phi[6,8$, (and hence as the dimensions of the real cohomology groups of the associated complex projective toric variety); see, for instance, 18, Section 5.2]. These Catalan and Narayana numbers are generalized by the numbers which appear in Theorems 1.1]and 1.2, respectively.

Theorem 1.2 is proved in Sections 3 and 4 by means of two bijections. The first maps the set of co-filtered chains of ideals in $\Phi^{+}$of length $m$ to the set of regions of $\mathrm{Cat}_{\Phi}^{m}$ in the fundamental chamber (Theorem [3.6). It specializes to the bijection of Shi [21, mentioned earlier, when $m=1$. The second maps this set of regions to the set of $W$-orbits of $T^{m}$ (see Lemma 4.1 and Theorem 4.2). Our proof in fact yields a refinement of Theorem 1.2 (see Corollaries 3.12 and 4.3 ). More precisely, 
it is shown that for any fixed $k$ with $1 \leq k \leq m$ and for corresponding chain $\mathcal{I}$, region $R$ and orbit $x$ under the bijections, indecomposable elements of $\mathcal{I}$ of rank $k$ (see Definition [3.8) correspond to facets of $R$ of the form $H_{\alpha, k}$ separating $R$ from $A_{\circ}$ and to facets of a certain simplex $\Sigma_{k}^{m}$ whose supporting hyperplanes contain the point of $\check{Q} \cap(m h+1) \overline{A_{\circ}}$ which represents the orbit $x$. The simplices $\Sigma_{k}^{m}$ are translated dilates of $\overline{A_{\circ}}$, one nested inside the other. In the case $m=1$, it turns out that the composite of our two bijections coincides with the one constructed in [5]. However, our motivation for constructing the second bijection comes from the paper by Shi 20, in which an element of the affine Weyl group is associated to each sign type of $\Phi$ in order to count the total number of sign types. The existence of such an element (for positive sign types) is generalized to arbitrary $m$ in Section 3 and plays an important role in our proof of Theorem [1.2. Section 2 includes notation and terminology related to root systems and their (affine) Weyl groups, as well as some basic facts that are needed in the following sections. In Section 5 we compute the numbers which appear in Theorem 1.2 in the cases of the classical root systems.

\section{Preliminaries}

In this section we introduce notation and terminology, and state a few elementary facts related to root systems and Weyl groups. We refer the reader to the text by Humphreys [11] for basic definitions and background.

Let $V$ be an $\ell$-dimensional Euclidean space with inner product ( , ). Given a hyperplane arrangement $\mathcal{A}$ in $V$, meaning a discrete set of affine subspaces of $V$ of codimension one, the regions of $\mathcal{A}$ are the connected components of the space obtained from $V$ by removing the hyperplanes in $\mathcal{A}$. Let $\Phi$ be an irreducible, crystallographic root system spanning $V$. We fix a positive system $\Phi^{+} \subseteq \Phi$ and the corresponding (ordered) set of simple roots $\Delta=\left(\sigma_{1}, \ldots, \sigma_{\ell}\right)$. Part (i) of the following lemma is well known, while part (ii) (which is stronger) follows from the results of Sommers in [22, Section 3] (see also [14 Lemma 2.3]).

Lemma 2.1. (i) If $\alpha_{1}, \alpha_{2}, \ldots, \alpha_{r} \in \Phi^{+}$with $r \geq 2$ and $\alpha=\alpha_{1}+\alpha_{2}+\cdots+\alpha_{r} \in \Phi^{+}$, then there exists $i$ with $1 \leq i \leq r$ such that $\alpha-\alpha_{i} \in \Phi^{+}$.

(ii) (14, 22]) If $\alpha_{1}, \alpha_{2}, \ldots, \alpha_{r} \in \Phi$ and $\alpha_{1}+\alpha_{2}+\cdots+\alpha_{r}=\alpha \in \Phi$, then $\alpha_{1}=\alpha$ or there exists $i$ with $2 \leq i \leq r$ such that $\alpha_{1}+\alpha_{i} \in \Phi \cup\{0\}$.

Proof. For reasons of self-containment we give a proof of part (ii). The statement is trivial for $r \leq 2$, so let $r \geq 3$ and proceed by induction on $r$. Clearly either $\alpha_{1}=\alpha$ or $\left(\alpha-\alpha_{1}, \alpha_{2}+\alpha_{3}+\cdots+\alpha_{r}\right)>0$, whence we conclude that $\left(\alpha_{1}, \alpha_{i}\right)<0$ or $\left(\alpha, \alpha_{i}\right)>0$ for some $i \geq 2$. In the former case it follows that $\alpha_{1}+\alpha_{i} \in \Phi \cup\{0\}$, as desired. In the latter case it follows that $\alpha-\alpha_{i} \in \Phi \cup\{0\}$ and hence, assuming $i=r$ without loss of generality, that either $\alpha_{1}+\alpha_{2}+\cdots+\alpha_{r-1}=\alpha-\alpha_{r} \in \Phi$ or $\alpha_{1}+\alpha_{2}+\cdots+\alpha_{r-2}=-\alpha_{r-1} \in \Phi$. Induction completes the proof in both cases.

We denote by $s_{1}, s_{2}, \ldots, s_{\ell}$, respectively, the reflections in the linear hyperpanes orthogonal to the elements of $\Delta$, called simple reflections. We let $\tilde{\alpha}$ be the highest root, $e_{1}, e_{2}, \ldots, e_{\ell}$ be the exponents and $h$ be the Coxeter number of $\Phi$, and we set $p=m h+1$, where $m$ is a fixed positive integer. The following fact can be checked directly, for instance, from the tables given in [11 Sections 3.18 and 4.9].

Lemma 2.2. If $\tilde{\alpha}=\sum_{i=1}^{\ell} c_{i} \sigma_{i}$, then $\sum_{i=1}^{\ell} c_{i}=h-1$. 
We denote by $\mathcal{A}_{\Phi}$ the Coxeter arrangement associated to $\Phi$, i.e., the collection of linear hyperplanes in $V$ which are orthogonal to the roots, and by $W$ the corresponding Weyl group, generated by the reflections in these hyperplanes. Thus $W$ is finite and minimally generated by the set of simple reflections; it leaves $\Phi$ invariant and acts simply transitively on the set of regions of $\mathcal{A}_{\Phi}$, called chambers. The fundamental chamber is the region defined by the inequalities $0<(\alpha, x)$ for $\alpha \in \Phi^{+}$. The set $Z(\Phi)$ of vectors $x \in \mathbb{R}^{\ell}$ satisfying $(\alpha, x) \in \mathbb{Z}$ for all $\alpha \in \Phi$ is the coweight lattice associated to $\Phi$. The coroot lattice $\check{Q}$ of $\Phi$ is the $\mathbb{Z}$-span of the set of coroots

$$
\Phi^{\vee}=\left\{\frac{2 \alpha}{(\alpha, \alpha)}: \alpha \in \Phi\right\} .
$$

Since $\Phi$ is crystallographic, we have $\check{Q}(\Phi) \subseteq Z(\Phi)$. The group $W$ acts on the lattice $\check{Q}$ and on its sublattice $p \check{Q}$; hence it also acts on the quotient $T^{m}=\check{Q} / p \check{Q}$. We denote by $O^{m}(\Phi)$ the set of orbits of the $W$-action on $T^{m}$, and we use the notation $T$ and $O(\Phi)$ when $m=1$.

For any real $k$ and $\alpha \in \Phi$ we denote by $H_{\alpha, k}$ the hyperplane in $V$ defined by the equation $(\alpha, x)=k$, and we set $H_{\alpha}=H_{\alpha, 0}$. We denote by $\widetilde{\mathcal{A}}_{\Phi}$ the affine Coxeter arrangement, which is the infinite hyperplane arrangement in $V$ consisting of the hyperplanes $H_{\alpha, k}$ for $\alpha \in \Phi$ and $k \in \mathbb{Z}$, and by $W_{a}$ the affine Weyl group, generated by the reflections in the hyperplanes of $\widetilde{\mathcal{A}}_{\Phi}$. The group $W_{a}$ is the semidirect product of $W$ and the translation group in $V$ corresponding to the coroot lattice $\check{Q}$, and is minimally generated by the set $\left\{s_{0}, s_{1}, \ldots, s_{\ell}\right\}$ of simple affine reflections, where $s_{0}$ is the reflection in the hyperplane $H_{\widetilde{\alpha}, 1}$. For $w \in W_{a}$ and $0 \leq i \leq \ell$, the reflection $s_{i}$ is a right descent of $w$ if $\ell\left(w s_{i}\right)<\ell(w)$, where $\ell(w)$ is the length of the shortest expression of $w$ as a product of simple affine reflections. The action of $W_{a}$ on $\widetilde{\mathcal{A}}_{\Phi}$ is determined by the following elementary lemma.

Lemma 2.3 ([11, Section 4.1]). For $w \in W, \alpha \in \Phi, \lambda \in \check{Q}$ and $k \in \mathbb{R}$ we have:

(i) $w H_{\alpha, k}=H_{w \alpha, k}$

(ii) $H_{\alpha, k}+\lambda=H_{\alpha, k+(\alpha, \lambda)}$.

The group $W_{a}$ acts simply transitively on the set of regions of $\widetilde{\mathcal{A}}_{\Phi}$ called alcoves. The fundamental alcove of $\widetilde{\mathcal{A}}_{\Phi}$ can be defined as

$$
A_{\circ}=\left\{x \in V: 0<\left(\sigma_{i}, x\right) \text { for } 1 \leq i \leq \ell \text { and }(\tilde{\alpha}, x)<1\right\} .
$$

Note that every alcove can be written as $w A_{\circ}$ for a unique $w \in W_{a}$. Moreover, given $\alpha \in \Phi^{+}$, there exists a unique integer $k$, denoted $k(w, \alpha)$, such that $k<$ $(\alpha, x)<k+1$ for all $x \in w A_{\circ}$. The next lemma is the main result of [19].

Lemma 2.4 (19, Theorem 5.2]). Let $k_{\alpha}$ be an integer for each $\alpha \in \Phi^{+}$. There exists $w \in W_{a}$ such that $k(w, \alpha)=k_{\alpha}$ for each $\alpha \in \Phi^{+}$if and only if

$$
k_{\alpha}+k_{\beta} \leq k_{\alpha+\beta} \leq k_{\alpha}+k_{\beta}+1
$$

for all $\alpha, \beta \in \Phi^{+}$with $\alpha+\beta \in \Phi^{+}$.

We say that two open regions in $V$ are separated by a hyperplane $H \in \widetilde{\mathcal{A}}_{\Phi}$ if they lie in different half-spaces relative to $H$. If $R$ is a region of a subarrangement of $\widetilde{\mathcal{A}}_{\Phi}$ or the closure of such a region (in particular, if $R$ is a chamber or an alcove), we refer to the hyperplanes of $\widetilde{\mathcal{A}}_{\Phi}$ which support facets of $R$ as the walls of $R$. Let 
$H_{0}=H_{\tilde{\alpha}, 1}$ and $H_{i}=H_{\sigma_{i}}$ for $1 \leq i \leq \ell$ be the walls of $A_{\circ}$. The next lemma follows, for instance, from the content of [11, Section 4.5].

Lemma 2.5. For $w \in W_{a}$ the number of walls of the alcove $w A_{\circ}$ separating $w A_{\circ}$ from $A_{\circ}$ is equal to the number of right descents of $w$.

\section{Ideals, Regions And the AfFine Weyl group}

In this section we generalize some of the work of Shi [20, 21] and Cellini and Papi [4] on regions of $\mathrm{Cat}_{\Phi}$ and order ideals in $\Phi^{+}$to regions of $\mathrm{Cat}_{\Phi}^{m}$ and cofiltered chains of ideals, and we prove part of Theorem 1.2. More specifically, we establish the equality of the numbers appearing in (i) and (iii) in the statement of the theorem.

Let $\mathcal{I}$ be a co-filtered chain of ideals

$$
\Phi^{+}=I_{0} \supseteq I_{1} \supseteq I_{2} \supseteq \cdots \supseteq I_{m}
$$

in $\Phi^{+}$of length $m$, so that (1.2) and (1.3) hold, where $J_{i}=\Phi^{+} \backslash I_{i}$. For any $\alpha \in \Phi^{+}$ we define

$$
k_{\alpha}(\mathcal{I})=\max \left\{k_{1}+k_{2}+\cdots+k_{r}: \alpha=\alpha_{1}+\alpha_{2}+\cdots+\alpha_{r} \text { with } \alpha_{i} \in I_{k_{i}} \text { for all } i\right\} .
$$

This quantity will play a major role in the sequel. Observe that $k_{\alpha}(\mathcal{I})=0$ if and only if $\alpha \in J_{1}$. Moreover, if $k_{\alpha}(\mathcal{I}) \geq 1$, then, by applying Lemma 2.1(ii) repeatedly, we can write $\alpha=\alpha_{1}+\alpha_{2}+\cdots+\alpha_{r}$ with $\alpha_{i} \in I_{k_{i}}, k_{\alpha}(\mathcal{I})=k_{1}+k_{2}+\cdots+k_{r}$ and $k_{i} \geq 1$ for all $i$. We begin with a few lemmas.

Lemma 3.1. If $\alpha=\alpha_{1}+\alpha_{2}+\cdots+\alpha_{r} \in \Phi^{+}$and $\alpha_{i} \in \Phi^{+}$for all $i$, then

$$
k_{\alpha}(\mathcal{I}) \geq \sum_{i=1}^{r} k_{\alpha_{i}}(\mathcal{I}) .
$$

Proof. This is clear from the definition.

Lemma 3.2. Let $\alpha \in \Phi^{+}$and $k_{\alpha}(\mathcal{I})=k$.

(i) We have $\alpha \in I_{\min \{k, m\}}$.

(ii) If $k>m$, then there exist $\beta, \gamma \in I_{1}$ with $\alpha=\beta+\gamma$ and $k=k_{\beta}(\mathcal{I})+k_{\gamma}(\mathcal{I})$. Moreover, we may choose $\beta$ so that $\beta \in I_{t}$, where $k_{\beta}(\mathcal{I})=t \leq m$.

Proof. Let $\alpha=\alpha_{1}+\alpha_{2}+\cdots+\alpha_{r}$ with $\alpha_{i} \in I_{k_{i}}$ for all $i$ and $k=k_{1}+k_{2}+\cdots+k_{r}$. We may assume that $k \geq 1$ and hence that $k_{i} \geq 1$ for all $i$. Conditions (1.2) and Lemma 2.1 (i) imply part (i) by induction on $r$. If $k>m$, then clearly $r \geq 2$, and by Lemma 2.1 (i) again there exists an index $j$ such that $\alpha-\alpha_{j} \in \Phi^{+}$. Let $\beta=\alpha_{j}$, $\gamma=\alpha-\alpha_{j}$ and $t=k_{j}$, and observe that $k_{\beta}(\mathcal{I}) \geq t$ and $k_{\gamma}(\mathcal{I}) \geq k-t$. Since $k_{\beta}(\mathcal{I})+k_{\gamma}(\mathcal{I}) \leq k$ by Lemma 3.1 we must have $k_{\beta}(\mathcal{I})=t$ and $k_{\gamma}(\mathcal{I})=k-t$. This proves (ii).

The next lemma generalizes Lemma 2.3 in 4 .

Lemma 3.3. If $\alpha, \beta, \alpha+\beta \in \Phi^{+}$and $a, b \geq 0$ are integers such that $k_{\alpha+\beta}(\mathcal{I}) \geq a+b$, then $k_{\alpha}(\mathcal{I}) \geq a$ or $k_{\beta}(\mathcal{I}) \geq b$. 
Proof. We proceed by induction on $k=k_{\alpha+\beta}(\mathcal{I})$. If $k \leq m$ then $\alpha+\beta \in I_{k}$ by Lemma 3.2 (i), and hence $\alpha+\beta \in I_{a+b}$. In view of (1.3) this implies that $\alpha \in I_{a}$ or $\beta \in I_{b}$, from which the result is clear. If $k>m$, by Lemma 3.2 (ii) we can write $\alpha+\beta=\eta+\theta$, where $\eta, \theta \in \Phi^{+}, k_{\eta}(\mathcal{I})=t, k_{\theta}(\mathcal{I})=k-t$ and $0<t<k$. If $\alpha=\eta$ and $\beta=\theta$ or vice versa, then $k_{\alpha}(\mathcal{I})+k_{\beta}(\mathcal{I})=k_{\eta}(\mathcal{I})+k_{\theta}(\mathcal{I})=k \geq a+b$, and the result is clear. If not, since

$$
(\alpha, \eta)+(\alpha, \theta)+(\beta, \eta)+(\beta, \theta)=(\alpha+\beta, \alpha+\beta)>0,
$$

one of the four summands on the left side must be positive. This implies that at least one of $\alpha-\eta=\theta-\beta$ and $\alpha-\theta=\eta-\beta$ is in $\Phi$. By exchanging the roles of $\eta$ and $\theta$, if necessary, we may assume that $\alpha-\eta \in \Phi$. By exchanging the roles of $\alpha$ and $\beta$ too, if necessary, we may assume further that $\alpha-\eta \in \Phi^{+}$. Let $\alpha=\eta+\mu$ with $\mu \in \Phi^{+}$, so that $\beta=\theta-\mu$. If $k_{\alpha}(\mathcal{I}) \geq a$, then there is nothing to prove. If $k_{\alpha}(\mathcal{I})<a$, then $k_{\eta}(\mathcal{I})+k_{\mu}(\mathcal{I})<a$ by Lemma 3.1 so that $k_{\mu}(\mathcal{I})<a-t$. Since $\theta=\beta+\mu$ and $k_{\theta}(\mathcal{I})=k-t<k$, it follows by induction that $k_{\beta}(\mathcal{I}) \geq k-a$ and hence that $k_{\beta}(\mathcal{I}) \geq b$, as desired.

Corollary 3.4. We have

$$
k_{\alpha}(\mathcal{I})+k_{\beta}(\mathcal{I}) \leq k_{\alpha+\beta}(\mathcal{I}) \leq k_{\alpha}(\mathcal{I})+k_{\beta}(\mathcal{I})+1
$$

whenever $\alpha, \beta, \alpha+\beta \in \Phi^{+}$.

Proof. The first inequality is a special case of Lemma 3.1 Letting $a=k_{\alpha}(\mathcal{I})+1$ and $b=k_{\alpha+\beta}(\mathcal{I})-a$, the second inequality follows from Lemma 3.3 in the case $b \geq 0$, and is obvious otherwise.

To define a region $R$ of $\mathrm{Cat}_{\Phi}^{m}$ in the fundamental chamber associated to $\mathcal{I}$, let $R_{\mathcal{I}}$ be the set of points $x \in V$ which satisfy

$$
\begin{array}{ll}
(\alpha, x)>k, & \text { if } \alpha \in I_{k}, \\
0<(\alpha, x)<k, & \text { if } \alpha \in J_{k},
\end{array}
$$

for $0 \leq k \leq m$.

Proposition 3.5. There exists $w \in W_{a}$ such that $k(w, \alpha)=k_{\alpha}(\mathcal{I})$ for $\alpha \in \Phi^{+}$. Moreover, $w A_{\circ} \subseteq R_{\mathcal{I}}$. In particular, $R_{\mathcal{I}}$ is nonempty.

Proof. The first statement follows from Lemma 2.4 and Corollary 3.4. For the second statement, let $\alpha \in \Phi^{+}$and $0 \leq k \leq m$. Part (i) of Lemma 3.2 implies that $k_{\alpha}(\mathcal{I}) \geq k$ if and only if $\alpha \in I_{k}$. Hence from the inequalities

$$
k_{\alpha}(\mathcal{I})<(\alpha, x)<k_{\alpha}(\mathcal{I})+1,
$$

which hold for $x \in w A_{\circ}$, we conclude that $w A_{\circ} \subseteq R_{\mathcal{I}}$.

Let $\psi$ be the map which assigns the set $R_{\mathcal{I}}$ to a co-filtered chain of ideals $\mathcal{I}$ in $\Phi^{+}$of length $m$. Conversely, given a region $R$ of $\mathrm{Cat}_{\Phi}^{m}$ in the fundamental chamber of $\mathcal{A}_{\Phi}$, let $\phi(R)$ be the sequence $\Phi^{+}=I_{0} \supseteq I_{1} \supseteq I_{2} \supseteq \cdots \supseteq I_{m}$, where $I_{k}$ is the set of $\alpha \in \Phi^{+}$for which $(\alpha, x)>k$ in $R$. The next theorem generalizes 21, Theorem 1.4].

Theorem 3.6. The map $\psi$ is a bijection from the set of co-filtered chains of ideals in $\Phi^{+}$of length $m$ to the set of regions of $\mathrm{Cat}_{\Phi}^{m}$ which lie in the fundamental chamber of $\mathcal{A}_{\Phi}$, and the map $\phi$ is its inverse. 


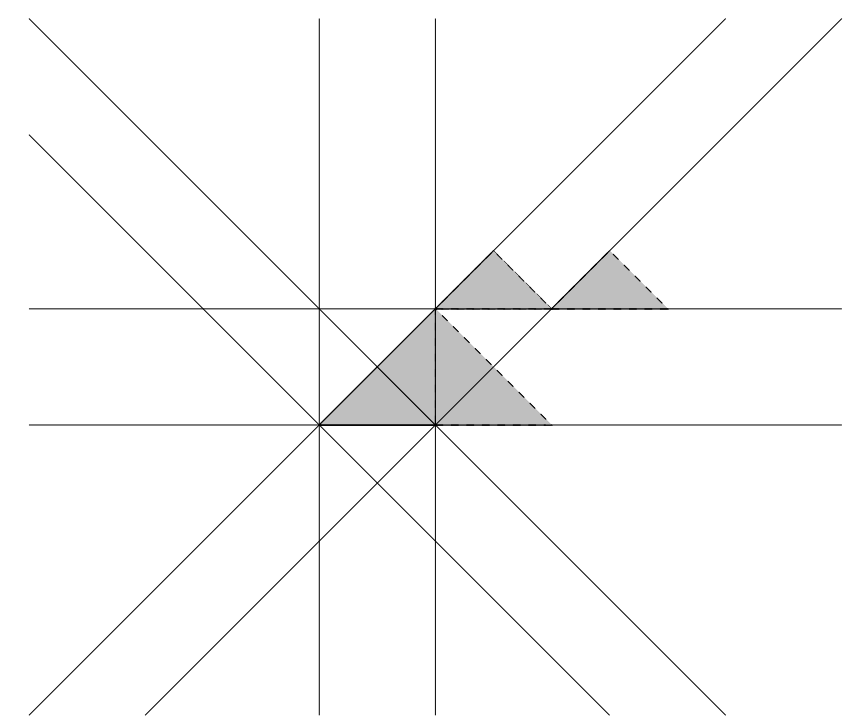

FiguRE 1. The representing alcoves of the regions in the fundamental chamber for $\Phi=B_{2}$ and $m=1$.

Proof. That the map $\psi$ is well defined follows from Proposition 3.5, which guarantees that the set $R_{\mathcal{I}}$ is nonempty. To check that $\phi$ is well defined, observe that if $R$ is a region of $\mathrm{Cat}_{\Phi}^{m}$ in the fundamental chamber of $\mathcal{A}_{\Phi}$ and if $(\alpha, x)>i$ and $(\beta, x)>j$ hold for $x \in R$, then $(\alpha+\beta, x)>i+j$ must hold for $x \in R$, so that $\phi(\mathcal{I})$ satisfies (1.2). Similarly, $\phi(\mathcal{I})$ satisfies (1.3). It is clear that $\psi$ and $\phi$ are inverses of each other.

Let $R=R_{\mathcal{I}}$ be a region of $\operatorname{Cat}_{\Phi}^{m}$ in the fundamental chamber of $\mathcal{A}_{\Phi}$, where $\mathcal{I}=\phi(R)$. Let $w_{R}$ denote the element of the affine Weyl group $W_{a}$ which is assigned to $\mathcal{I}$ in Proposition 3.5. The following proposition implies that $w_{R} A_{\circ}$ is the alcove in $R$ which is closest to $A_{\circ}$; see Figures 1 and $2(\S 4)$. In the special case $m=1$ the existence of such an alcove was established by Shi [20, Proposition 7.2] for all regions of the arrangement in $V$ consisting of the hyperplanes $H_{\alpha}$ and $H_{\alpha, 1}$ for $\alpha \in \Phi^{+}$.

Proposition 3.7. Let $R$ be a region of $\mathrm{Cat}_{\Phi}^{m}$ in the fundamental chamber of $\mathcal{A}_{\Phi}$. The element $w_{R}$ is the unique $w \in W_{a}$ such that $w A_{\circ} \subseteq R$ and whenever $\alpha \in \Phi^{+}$, $k \in \mathbb{Z}$ and $(\alpha, x)<k$ for some $x \in R$ we have $(\alpha, x)<k$ for all $x \in w A_{\circ}$.

Proof. Suppose that $\alpha \in \Phi^{+}$, and $(\alpha, x)<k$ holds for some $x \in R=R_{\mathcal{I}}$ and $k \in \mathbb{Z}$. To show that $(\alpha, x)<k$ holds for $x \in w_{R} A_{\circ}$ it suffices to show that $k_{\alpha}(\mathcal{I})<k$. Let us write $\alpha=\alpha_{1}+\alpha_{2}+\cdots+\alpha_{r}$ with $\alpha_{i} \in I_{k_{i}}$ for all $i$ and $k_{1}+k_{2}+\cdots+k_{r}=k_{\alpha}(\mathcal{I})$. We have $\left(\alpha_{i}, x\right)>k_{i}$ for all $x \in R$, and hence $(\alpha, x)>k_{\alpha}(\mathcal{I})$ for all such $x$. Thus if $(\alpha, x)<k$ for some $x \in R$, we must have $k_{\alpha}(\mathcal{I})<k$. Uniqueness is clear.

We now introduce the elements of $\Phi^{+}$which will play the role that the minimal elements of the ideal $I$ play in the case $m=1$. 
Definition 3.8. Given $1 \leq k \leq m$, a root $\alpha \in \Phi^{+}$is indecomposable of rank $k$ with respect to $\mathcal{I}$ if $\alpha \in I_{k}$ and

(i) $k_{\alpha}(\mathcal{I})=k$,

(ii) it is not possible to write $\alpha=\beta+\gamma$ with $\beta \in I_{i}$ and $\gamma \in I_{j}$ for indices $i, j \geq 0$ with $i+j=k$, and

(iii) if $\alpha+\beta \in I_{t}$ for some $\beta \in \Phi^{+}$and $t \leq m$ and if $k_{\alpha+\beta}(\mathcal{I})=t$, then $\beta \in I_{t-k}$.

Observe that, by part (i) of Lemma 3.2. the assumption $\alpha \in I_{k}$ in this definition is actually implied by condition (i). For $k=m$ the definition is equivalent to the one proposed in Section 1 as the following lemma shows.

Lemma 3.9. A positive root $\alpha$ is indecomposable of rank $m$ with respect to $\mathcal{I}$ if and only if $\alpha \in I_{m}$ and it is not possible to write $\alpha=\beta+\gamma$ with $\beta \in I_{i}$ and $\gamma \in I_{j}$ for indices $i, j \geq 0$ with $i+j=m$.

Proof. Suppose that $\alpha \in I_{m}$ satisfies the condition in the statement of the lemma. Since condition (iii) in Definition 3.8 is trivial for $k=m$, to show that $\alpha$ is indecomposable of rank $m$ it suffices to show that $k_{\alpha}(\mathcal{I})=m$. Indeed, if $k_{\alpha}(\mathcal{I})=k>m$, then, by part (ii) of Lemma 3.2 we can write $\alpha=\beta+\gamma$ with $\beta \in I_{t}$ for some $1 \leq t \leq m, \gamma \in I_{1}$ and $k_{\gamma}(\mathcal{I})=k-t$. Then $k_{\gamma}(\mathcal{I})>m-t$, and part (i) of the same lemma implies that $\gamma \in I_{m-t}$, contrary to the hypothesis. The converse is trivial.

Lemma 3.10. Suppose that $\alpha$ is indecomposable with respect to $\mathcal{I}$.

(i) We have $k_{\alpha}(\mathcal{I})=k_{\beta}(\mathcal{I})+k_{\gamma}(\mathcal{I})+1$ whenever $\alpha=\beta+\gamma$ with $\beta, \gamma \in \Phi^{+}$.

(ii) We have $k_{\alpha}(\mathcal{I})+k_{\beta}(\mathcal{I})=k_{\alpha+\beta}(\mathcal{I})$ whenever $\beta, \alpha+\beta \in \Phi^{+}$.

Proof. Let $k_{\alpha}(\mathcal{I})=k$, so that $\alpha \in I_{k}$.

(i) Suppose that $\alpha=\beta+\gamma$ with $\beta, \gamma \in \Phi^{+}$, and let $k_{\beta}(\mathcal{I})=i$ and $k_{\gamma}(\mathcal{I})=j$. By Lemma 3.2 (i) we have $\beta \in I_{i}$ and $\gamma \in I_{j}$, so condition (ii) in Definition 3.8 does not allow that $k=i+j$. By Corollary 3.4 we must have $k=i+j+1$.

(ii) Suppose that $\beta, \alpha+\beta \in \Phi^{+}$and write $\alpha+\beta=\alpha_{1}+\alpha_{2}+\cdots+\alpha_{r}$ with $\alpha_{i} \in I_{k_{i}}$ for all $i$ and $k_{1}+k_{2}+\cdots+k_{r}=k_{\alpha+\beta}(\mathcal{I})$. Letting $t=k_{\alpha+\beta}(\mathcal{I})$, by Corollary 3.4 it suffices to show that $k_{\beta}(\mathcal{I}) \geq t-k$. If $r=1$, then $\alpha+\beta \in I_{t}$, and condition (iii) in Definition 3.8 implies that $\beta \in I_{t-k}$, so the desired inequality is clear. Suppose $r \geq 2$. We have $-\alpha+\alpha_{1}+\cdots+\alpha_{r}=\beta \in \Phi$, and hence $-\alpha+\alpha_{i} \in \Phi \cup\{0\}$ for some $i$ by Lemma 2.1 (ii), say for $i=1$. Thus either $\alpha=\alpha_{1}$ or $\alpha=\alpha_{1}+\mu$ or $\alpha_{1}=\alpha+\mu$ with $\mu \in \Phi^{+}$. In the first case we have $\beta=\alpha_{2}+\cdots+\alpha_{r}$, and hence $k_{\beta}(\mathcal{I})=t-k_{1}=t-k$. In the second case we have $\beta+\mu=\alpha_{2}+\cdots+\alpha_{r}$, and hence $k_{\beta+\mu}(\mathcal{I})=k_{2}+\cdots+k_{r}=t-k_{1}$. Since $\alpha$ is indecomposable, we have $k=k_{\alpha}(\mathcal{I})=k_{\alpha_{1}}(\mathcal{I})+k_{\mu}(\mathcal{I})+1=k_{1}+k_{\mu}(\mathcal{I})+1$ by Lemma 3.10 (i), and hence

$$
k_{\beta}(\mathcal{I}) \geq k_{\beta+\mu}(\mathcal{I})-k_{\mu}(\mathcal{I})-1=k_{\beta+\mu}(\mathcal{I})+k_{1}-k=t-k .
$$

In the last case we have $k_{\mu}(\mathcal{I}) \geq k_{1}-k$ by the case $r=1$ treated earlier and $\beta=\mu+\alpha_{2}+\cdots+\alpha_{r}$, so

$$
k_{\beta}(\mathcal{I}) \geq k_{\mu}(\mathcal{I})+k_{2}+\cdots+k_{r} \geq t-k .
$$

This completes the proof.

The following theorem explains the connection between indecomposable elements of $\mathcal{I}$ and separating walls of $R_{\mathcal{I}}$. Recall from the proof of Proposition 3.7 that $(\alpha, x)>k_{\alpha}(\mathcal{I})$ holds for all $\alpha \in \Phi^{+}$and $x \in R_{\mathcal{I}}$. 
Theorem 3.11. If $\mathcal{I}$ is a co-filtered chain of ideals in $\Phi^{+}$of length $m$ with corresponding region $R=R_{\mathcal{I}}$ and $1 \leq k \leq m$, then the following sets are equal:

(i) the set of indecomposable roots $\alpha \in \Phi^{+}$with respect to $\mathcal{I}$ of rank $k$,

(ii) the set of $\alpha \in \Phi^{+}$such that $H_{\alpha, k}$ is a wall of $R$ separating $R$ from $A_{\circ}$, and

(iii) the set of $\alpha \in \Phi^{+}$such that $H_{\alpha, k}$ is a wall of $w_{R} A_{\circ}$ separating $w_{R} A_{\circ}$ from $A_{\circ}$.

Proof. Let $\Phi^{+}=I_{0} \supseteq I_{1} \supseteq I_{2} \supseteq \cdots \supseteq I_{m}$ be the chain $\mathcal{I}$, as usual. Let $w=w_{R}$ and let $F_{k}(\mathcal{I}), F_{k}(R)$ and $F_{k}(w)$ denote the sets refered to in (i), (ii) and (iii), respectively. To prove that these sets coincide, we will show that $F_{k}(R) \subseteq F_{k}(\mathcal{I}) \subseteq$ $F_{k}(w) \subseteq F_{k}(R)$.

Suppose that $\alpha \in F_{k}(R)$. Since $(\alpha, x)>k$ holds for $x \in R$, we have $\alpha \in I_{k}$. To show that $\alpha$ is indecomposable of rank $k$ with respect to $\mathcal{I}$, we need to check that the conditions in Definition 3.8 are satisfied. We first claim that it is not possible to write $\alpha=\beta+\gamma$ with $\beta, \gamma \in \Phi^{+}$satisfying $k_{\beta}(\mathcal{I}) \geq i$ and $k_{\gamma}(\mathcal{I}) \geq j$ for some $i, j \geq 0$ with $i+j=k$. Indeed, if that were the case, then $(\beta, x)>i$ and $(\gamma, x)>j$ would be valid for $x \in R$. Since these inequalities imply the inequality $(\alpha, x)>k$, the hyperplane $H_{\alpha, k}$ would not support a facet of $R$. Clearly the claim implies condition (ii). In view of Lemma 3.2 (ii) it also implies that $k_{\alpha}(\mathcal{I}) \leq m$, so we have $k \leq k_{\alpha}(\mathcal{I}) \leq m$. If $k=m$, then we must have $k_{\alpha}(\mathcal{I})=m$. If $k<m$, then $(\alpha, x)<k+1$ holds for $x \in R$, and hence $\alpha \in J_{k+1}$. Lemma 3.2 (i) then forces that $k_{\alpha}(\mathcal{I})=k$, and this verifies condition (i). Similarly, to verify (iii) suppose that $\alpha+\beta \in I_{t}$ for some $\beta \in \Phi^{+}$and $k_{\alpha+\beta}(\mathcal{I})=t$. Since $(\alpha, x)>k$ is implied by the inequalities $(\alpha+\beta, x)>t$ and $(\beta, x)<t-k$ and the first of these is valid for $x \in R$, the second cannot be valid for all such $x$. This means that $\beta \in I_{t-k}$.

Next suppose that $\alpha \in F_{k}(\mathcal{I})$, meaning that $\alpha$ is indecomposable of rank $k$ with respect to $\mathcal{I}$. We have $(\alpha, x)>k$ for all $x \in R$ and hence all $x \in w A_{\circ}$. Recall that $k(w, \beta)=k_{\beta}(\mathcal{I})$ for all $\beta \in \Phi^{+}$. To show that $H_{\alpha, k}$ is a wall of $w A_{\circ}$, it suffices to show that there exists a $w^{\prime} \in W_{a}$ with $k\left(w^{\prime}, \beta\right)=k_{\beta}$ for all $\beta \in \Phi^{+}$, where

$$
k_{\beta}= \begin{cases}k_{\beta}(\mathcal{I}), & \text { if } \beta \neq \alpha, \\ k_{\alpha}(\mathcal{I})-1, & \text { if } \beta=\alpha .\end{cases}
$$

In view of Lemma 2.4 and Corollary 3.4, this amounts to proving that $k_{\alpha}(\mathcal{I})=$ $k_{\beta}(\mathcal{I})+k_{\gamma}(\mathcal{I})+1$ whenever $\alpha=\beta+\gamma$ with $\beta, \gamma \in \Phi^{+}$, and that $k_{\alpha}(\mathcal{I})+k_{\beta}(\mathcal{I})=$ $k_{\alpha+\beta}(\mathcal{I})$ whenever $\beta, \alpha+\beta \in \Phi^{+}$. This is precisely the content of Lemma 3.10

Finally, suppose that $\alpha \in F_{k}(w)$. If $w^{\prime} A_{\circ}$ is the alcove of $\widetilde{\mathcal{A}}_{\Phi}$ which is the reflection of $w A_{\circ}$ on its wall $H_{\alpha, k}$, then $(\alpha, x)<k$ holds for all $x \in w^{\prime} A_{\circ}$. Proposition 3.7 implies that $w^{\prime} A_{\circ}$ is not contained in $R$. Hence $H_{\alpha, k}$ must be a wall of $R$; in other words, $\alpha \in F_{k}(R)$.

We denote by $W_{\text {Cat }}^{m}$ the subset of $W_{a}$ consisting of the elements $w_{R}$ for the regions $R$ of $\mathrm{Cat}_{\Phi}^{m}$ in the fundamental chamber of $\mathcal{A}_{\Phi}$, and abbreviate this set as $W_{\text {Cat }}$ in the case $m=1$.

Corollary 3.12. For any nonnegative integers $i_{1}, i_{2}, \ldots, i_{m}$ the following are equal:

(i) the number of co-filtered chains of ideals in $\Phi^{+}$of length $m$ having $i_{k}$ indecomposable elements of rank $k$ for each $1 \leq k \leq m$, 
(ii) the number of regions $R$ of $\mathrm{Cat}_{\Phi}^{m}$ in the fundamental chamber of $\mathcal{A}_{\Phi}$ such that $i_{k}$ walls of $R$ of the form $H_{\alpha, k}$ separate $R$ from $A_{\circ}$ for each $1 \leq k \leq m$, and

(iii) the number of $w \in W_{\mathrm{Cat}}^{m}$ such that $i_{k}$ walls of $w A_{\circ}$ of the form $H_{\alpha, k}$ separate $w A_{\circ}$ from $A_{\circ}$ for each $1 \leq k \leq m$.

Proof. Combine Theorems 3.6 and 3.11

The following corollary is immediate.

Corollary 3.13. For any nonnegative integer $i$ the quantities which appear in (i) and (iii) in the statement of Theorem 1.2 are both equal to the number of $w \in W_{\text {Cat }}^{m}$ such that $i$ walls of $w A_{\circ}$ of the form $H_{\alpha, m}$ separate $w A_{\circ}$ from $A_{\circ}$.

We now consider the special case $m=1$. A co-filtered chain of ideals $\mathcal{I}$ of length $m$ in this case is simply a single ideal $I$ in $\Phi^{+}$and, by Lemma 3.9] the indecomposable elements of $\mathcal{I}$ are exactly the minimal elements of $I$. These elements form an antichain in $\Phi^{+}$, meaning a subset of pairwise incomparable elements. Conversely, any antichain $A \subseteq \Phi^{+}$defines the ideal $I_{A}$ which consists of all $\beta \in \Phi^{+}$ such that $\beta \geq \alpha$ for some $\alpha \in A$. The set of minimal elements of $I_{A}$ is equal to $A$, and the map which assigns $I_{A}$ to $A$ is a bijection from the set of antichains to the set of ideals in $\Phi^{+}$.

Corollary 3.14. For any nonnegative integer $i$ the following are equal:

(i) the number of $i$-element antichains in the root poset of $\Phi$,

(ii) the number of regions $R$ of $\mathrm{Cat}_{\Phi}$ in the fundamental chamber of $\mathcal{A}_{\Phi}$ such that $i$ walls of $R$ separate $R$ from $A_{\circ}$,

(iii) the number of $w \in W_{\text {Cat }}$ such that $i$ walls of $w A_{\circ}$ separate $w A_{\circ}$ from $A_{\circ}$, and

(iv) the number of elements $w \in W_{\text {Cat }}$ having $i$ right descents.

Proof. This follows from the case $m=1$ of Corollary 3.12 and Lemma 2.5

Observe that the region of $\mathrm{Cat}_{\Phi}$ which corresponds to the ideal $I_{A}$ under the bijection $\psi$ of Theorem 3.6 is the set $R_{A}$ of points $x$ in $V$ which satisfy

$$
\begin{array}{ll}
(\beta, x)>1, & \text { if } \beta \in I_{A}, \\
0<(\beta, x)<1, & \text { if } \beta \in \Phi^{+} \backslash I_{A} .
\end{array}
$$

By Theorem [3.6, the map which sends an antichain $A$ to the set $R_{A}$ is a bijection from the set of antichains in $\Phi^{+}$to the set of regions of Cat Can $_{\text {in }}$ the fundamental chamber of $\mathcal{A}_{\Phi}$. This bijection was first established and exploited by Shi 21. In view of Theorem 3.11 (applied for $k=m=1$ ), its inverse sends a region $R$ to the set of $\alpha \in \Phi^{+}$such that $H_{\alpha, 1}$ is a wall of $R$ and $(\alpha, x)>1$ holds for $x \in R$.

\section{Proof of the main theorem}

In this section we complete the proof of Theorem 1.2 (see Corollary 4.4). We begin with some more notation and known facts on the action of $W$ on $T^{m}$.

By the reflection in $W$ corresponding to a hyperplane $H_{\alpha, k}$ we mean the reflection in the linear hyperplane $H_{\alpha}$. We let $p=m h+1$, as in Section 2 and denote by $D_{\Phi}^{m}$ the set $\breve{Q} \cap p \overline{A_{\circ}}$. The following lemma, which is part of [10, Lemma 7.4.1], implies that $D_{\Phi}^{m}$ is a set of representatives for the orbits of the $W$-action on $T^{m}$. 


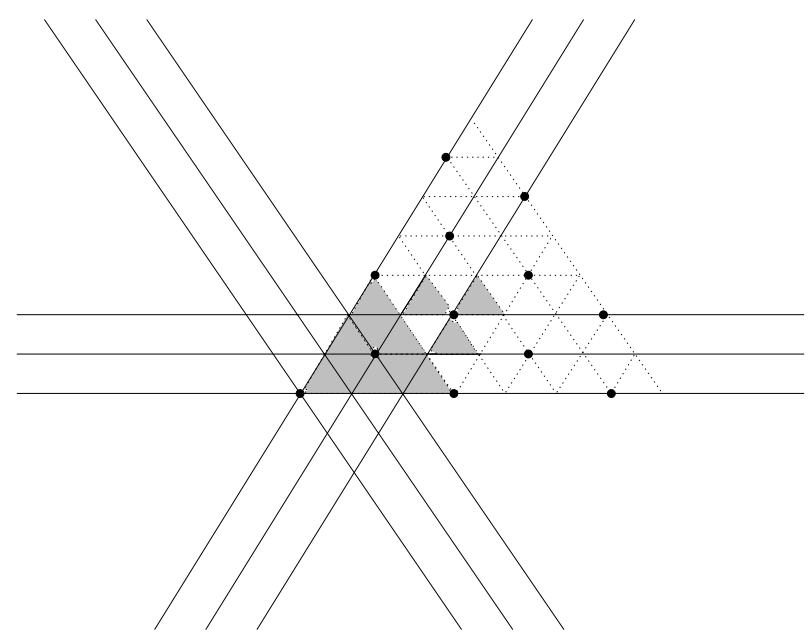

Figure 2. The representing alcoves of the regions in the fundamental chamber and the simplex $p \overline{A_{\circ}}$ for $\Phi=A_{2}$ and $m=2$.

Lemma 4.1. The natural inclusion map from $D_{\Phi}^{m}$ to the set $O^{m}(\Phi)$ of orbits of the $W$-action on $T^{m}$ is a bijection.

Moreover, if $y \in D_{\Phi}^{m}$, then the stabilizer of $y$ with respect to the $W$-action on $T^{m}$ is the subgroup of $W$ generated by the reflections corresponding to the walls of $p \overline{A_{\circ}}$ which contain $y$. In particular, $r(y)$ is equal to the number of walls of $p \overline{A_{\circ}}$ which contain $y$.

Our objective is to define a bijection $\rho: W_{\text {Cat }}^{m} \rightarrow D_{\Phi}^{m}$ such that for $w \in W_{\text {Cat }}^{m}$, the number of walls of $w A_{\circ}$ of the form $H_{\alpha, m}$ which separate $w A_{\circ}$ from $A_{\circ}$ is equal to the number of walls of $p \overline{A_{\circ}}$ which contain $\rho(w)$. Let $R_{f}$ be the region of $\mathrm{Cat}_{\Phi}^{m}$ defined by the inequalities $m<\left(\sigma_{i}, x\right)$ for $1 \leq i \leq \ell$. Let $w_{f}=w_{R_{f}}$ be the unique element $w$ of $W_{\text {Cat }}^{m}$ such that $w A_{\circ} \subseteq R_{f}$. Note that $w_{f}$ is not necessarily a translation in $V$ (see, for instance, Figure 1). We define the map $\rho: W_{\text {Cat }}^{m} \rightarrow \check{Q}$ by

$$
\rho(w)=\left(w_{f} w^{-1}\right) \cdot 0
$$

for $w \in W_{\text {Cat }}^{m}$. Observe that, by Lemma 2.2 the alcove $w_{f} A_{\circ}$ can be described explicitly as the open simplex in $V$ defined by the linear inequalities $m<\left(\sigma_{i}, x\right)$ for $1 \leq i \leq \ell$ and $(\tilde{\alpha}, x)<m h-m+1$. For any $0 \leq k \leq m$ we define the simplex

$$
\Sigma_{k}^{m}=\left\{x \in V: m-k \leq\left(\sigma_{i}, x\right) \text { for } 1 \leq i \leq \ell \text { and }(\tilde{\alpha}, x) \leq m h-m+k+1\right\},
$$

so that $\Sigma_{k}^{m}$ reduces to $w_{f} \overline{A_{\circ}}$ and $p \overline{A_{\circ}}$ for $k=0$ and $k=m$, respectively. For any $\ell$ dimensional simplex $\Sigma$ in $V$ bounded by hyperplanes $H_{\alpha, k}$ in $\widetilde{\mathcal{A}}_{\Phi}$ with $\alpha \in \Delta \cup\{\tilde{\alpha}\}$ we denote by $H(\Sigma, i)$ the wall of $\Sigma$ orthogonal to $\tilde{\alpha}$ or $\sigma_{i}$, if $i=0$ or $i>0$, respectively. We write $H(w, i)$ instead of $H\left(w \overline{A_{\circ}}, i\right)$ for $w \in W_{a}$. The following theorem is the main result of this section. The reader is invited to verify the statement of the theorem in the case pictured in Figure2.

Theorem 4.2. The map $\rho$ is a bijection from $W_{\mathrm{Cat}}^{m}$ to $D_{\Phi}^{m}$. Moreover, for any $w \in W_{\mathrm{Cat}}^{m}, 1 \leq k \leq m$ and $0 \leq i \leq \ell$, the point $\rho(w)$ lies on the wall $H\left(\Sigma_{k}^{m}, i\right)$ if and only if the wall $\left(w w_{f}^{-1}\right) H\left(w_{f}, i\right)$ of $w A_{\circ}$ is of the form $H_{\alpha, k}$ and separates $w A_{\circ}$ from $A_{\circ}$. 
Proof. First we will check that $\rho$ is injective. Indeed, if $\rho\left(w_{1}\right)=\rho\left(w_{2}\right)$, with $w_{1}, w_{2} \in W_{\text {Cat }}^{m}$, then $w_{2} w_{1}^{-1}$ fixes the origin, and hence $w_{2} w_{1}^{-1} \in W$. Moreover, $w_{2} w_{1}^{-1}$ carries $w_{1} A_{\circ}$ to $w_{2} A_{\circ}$, and both alcoves lie in the fundamental chamber of $\mathcal{A}_{\Phi}$. Since $W$ acts simply transitively on the chambers of $\mathcal{A}_{\Phi}, w_{2} w_{1}^{-1}$ must be the identity; that is, $w_{1}=w_{2}$.

We next check that $\rho(w) \in D_{\Phi}^{m}$ for $w \in W_{\text {Cat }}^{m}$, and prove the second statement in the theorem. Clearly $\rho(w) \in \check{Q}$, so for the first claim it suffices to show that $\rho(w) \in p \overline{A_{\circ}}$. Let us write $w_{f} w^{-1}=t_{\lambda} u$, where $\lambda \in \check{Q}, t_{\lambda}$ is the translation map in $V$ which sends $x$ to $x+\lambda$, and $u \in W$, so that $\rho(w)=\lambda$. Let $0 \leq i \leq \ell, 1 \leq k \leq m$, and let $H$ be the wall $\left(w w_{f}^{-1}\right) H\left(w_{f}, i\right)$ of $w A_{\circ}$. We need to show (i) that

$$
\begin{array}{ll}
(\lambda, \tilde{\alpha}) \leq m h+1, & \text { if } i=0, \\
\left(\lambda, \sigma_{i}\right) \geq 0, & \text { if } 1 \leq i \leq \ell,
\end{array}
$$

and (ii) that $H=H_{\alpha, k}$ for some $\alpha \in \Phi^{+}$with $H$ separating $w A_{\circ}$ from $A_{\circ}$ if and only if

$$
\begin{array}{ll}
(\lambda, \tilde{\alpha})=m h-m+k+1, & \text { if } i=0, \\
\left(\lambda, \sigma_{i}\right)=m-k, & \text { if } 1 \leq i \leq \ell .
\end{array}
$$

Let $H=H_{\alpha, r}$ for some $\alpha \in \Phi^{+}$and nonnegative integer $r$. Observe first that $H$ separates $w A_{\circ}$ from $A_{\circ}$ if and only if $H\left(w_{f}, i\right)=\left(w_{f} w^{-1}\right) H$ separates $w_{f} A_{\circ}$ from $\left(w_{f} w^{-1}\right) A_{\circ}=\left(t_{\lambda} u\right) A_{\circ}$ or, equivalently, if and only if

$$
\begin{array}{ll}
(u x+\lambda, \tilde{\alpha})>m h-m+1, & \text { if } i=0, \\
\left(u x+\lambda, \sigma_{i}\right)<m, & \text { if } 1 \leq i \leq \ell,
\end{array}
$$

for all $x \in A_{\circ}$. Next observe that $0<(\beta, x)<1$ for all $x \in A_{\circ}$ and $\beta \in \Phi^{+}$, and hence that $-1<(\beta, u x)<1$ holds for all such $x$ and $\beta$. In view of Lemma 2.3, the fact that $t_{\lambda} u H_{\alpha, r}=w_{f} w^{-1} H=H\left(w_{f}, i\right)$ implies that

$$
H_{u \alpha, r+(u \alpha, \lambda)}= \begin{cases}H_{\tilde{\alpha}, m h-m+1}, & \text { if } i=0 \\ H_{\sigma_{i}, m}, & \text { if } 1 \leq i \leq \ell .\end{cases}
$$

To prove (4.1), suppose $i=0$ first. It follows from (4.4) that either $u \alpha=\tilde{\alpha}$ and $r+(u \alpha, \lambda)=m h-m+1$, or $u \alpha=-\tilde{\alpha}$ and $r+(u \alpha, \lambda)=-m h+m-1$. Therefore $(\lambda, \tilde{\alpha})=m h-m-r+1$ or $m h-m+r+1$. Thus to prove that $(\lambda, \tilde{\alpha}) \leq m h+1$ it suffices to show that we have $r \leq m$ in the second case. Indeed, suppose that $(\lambda, \tilde{\alpha})=m h-m+r+1$. We may assume that $r \geq 1$. From the statement in (4.3) we conclude that $H=H_{\alpha, r}$ separates $w A_{\circ}$ from $A_{\circ}$. Since $H$ is a facet of a region of $\mathrm{Cat}_{\Phi}^{m}$ in the fundamental chamber of $\mathcal{A}_{\Phi}$ (Theorem 3.11), we must have $r \leq m$. The case $1 \leq i \leq \ell$ is similar. From (4.4) we get that either $u \alpha=\sigma_{i}$ and $r+(u \alpha, \lambda)=m$, or $u \alpha=-\sigma_{i}$ and $r+(u \alpha, \lambda)=-m$. Therefore $\left(\lambda, \sigma_{i}\right)=m-r$ or $m+r$. If $\left(\lambda, \sigma_{i}\right)=m-r$ and $r \geq 1$, it follows again from the statement in (4.3) that $H=H_{\alpha, r}$ separates $w A_{\circ}$ from $A_{\circ}$, and hence $r \leq m$, as before. Thus (4.1) holds, which means that $\rho(w) \in p \overline{A_{\circ}}$.

We now prove the second claim. Suppose first that $H=H_{\alpha, k}$ for some $\alpha \in \Phi^{+}$, and that $H$ separates $w A_{\circ}$ from $A_{\circ}$. Then we have $k \geq 1$, and the analysis we just went through with $r$ replaced by $k$ shows that $(\lambda, \tilde{\alpha})=m h-m-k+1$ or $m h-m+k+1$ if $i=0$, and that $\left(\lambda, \sigma_{i}\right)=m-k$ or $m+k$ if $1 \leq i \leq \ell$. In view of (4.3) and the inequality $-1<(u x, \beta)<1$ for $x \in A_{\circ}$ and $\beta \in \Phi^{+}$, equation (4.2) follows. Conversely, suppose that (4.2) holds for some $1 \leq k \leq m$. Then (4.3) is 
valid, and hence $H$ separates $w A_{\circ}$ from $A_{\circ}$. Letting $H=H_{\alpha, r}$ for some $r \geq 1$, we deduce (4.4), as before, and that $(\lambda, \tilde{\alpha})=m h-m-r+1$ or $m h-m+r+1$ if $i=0$, while $\left(\lambda, \sigma_{i}\right)=m-r$ or $m+r$ if $1 \leq i \leq \ell$. It follows from (4.2) that $r=k$.

Finally, note that $W_{\text {Cat }}^{m}$ and $D_{\Phi}^{m}$ are equinumerous by Theorem 1.1. Since the map $\rho: W_{\text {Cat }}^{m} \rightarrow D_{\Phi}^{m}$ is injective, it has to be a bijection.

Corollary 4.3. For any nonnegative integers $i_{1}, i_{2}, \ldots, i_{m}$ each of the quantities which appear in the statement of Corollary 3.12 is equal to the number of points in $D_{\Phi}^{m}$ which lie in $i_{k}$ walls of $\Sigma_{k}^{m}$ for all $1 \leq k \leq m$.

Proof. Indeed, it follows from Theorem 4.2 that the number of $w \in W_{\text {Cat }}^{m}$ such that $i_{k}$ walls of $w A_{\circ}$ of the form $H_{\alpha, k}$ separate $w A_{\circ}$ from $A_{\circ}$ for $1 \leq k \leq m$ is equal to the number of points $x \in D_{\Phi}^{m}$ such that $x$ lies in $i_{k}$ walls of $\Sigma_{k}^{m}$ for $1 \leq k \leq m$.

The following corollary implies Theorem 1.2 .

Corollary 4.4. For any nonnegative integer $i$ each of the quantities which appear in the statement of Theorem 1.2 is equal to

(i) the number of points in $D_{\Phi}^{m}$ which lie in $i$ walls of $p \overline{A_{\circ}}$, and

(ii) the number of $w \in W_{\mathrm{Cat}}^{m}$ such that $i$ walls of $w A_{\circ}$ of the form $H_{\alpha, m}$ separate $w A_{\circ}$ from $A_{\circ}$.

Proof. By Lemma 4.1, the number of orbits $x \in O^{m}(\Phi)$ with rank $r(x)=i$ is equal to the number of points in $D_{\Phi}^{m}$ which lie in $i$ walls of $p \overline{A_{\circ}}$. The statement now follows by specializing Corollary 4.3 and recalling that $\Sigma_{k}^{m}=p \overline{A_{\circ}}$ for $k=m$.

Remark 4.5. For $i=0$, the number which appears in Theorem 1.2 is equal to

$$
\prod_{i=1}^{\ell} \frac{e_{i}+(m-1) h+1}{e_{i}+1}
$$

Indeed, in view of the fact that $\check{Q}(\Phi) \subseteq Z(\Phi)$, the number of points in $D_{\Phi}$ lying in no wall of $p \overline{A_{\circ}}$ is equal to the cardinality of $\breve{Q} \cap \Sigma_{m-1}^{m}$. By Lemma 2.2, the simplex $\Sigma_{m-1}^{m}$ is a translate of $(p-h) \overline{A_{\circ}}$ by an element of the coweight lattice $Z(\Phi)$. It follows, for instance, from [5, Lemma 1] and the remark following this lemma that there exists a $w \in W_{a}$ such that $(p-h) \overline{A_{\circ}}=w\left(\Sigma_{m-1}^{m}\right)$. Thus $\check{Q} \cap \Sigma_{m-1}^{m}$ is equinumerous to $\check{Q} \cap(p-h) \overline{A_{\circ}}$. Our claim then follows from Corollary 4.4, Lemma 4.1 and Theorem 1.1 where $m$ is replaced by $m-1$.

\section{The Classical TYPES}

In this section we compute the numbers which appear in Theorem 1.2 in the cases of the classical root systems, as follows.

Proposition 5.1. Let $\Phi$ be a root system, $W$ its Weyl group, and let $T^{m}$ be as in Theorem 1.2. The number of $W$-orbits $x$ of $T^{m}$ with $r(x)=n-k$ is equal to

$$
\begin{array}{lr}
\frac{1}{k}\left(\begin{array}{l}
n-1 \\
k-1
\end{array}\right)\left(\begin{array}{c}
m n \\
k-1
\end{array}\right), & \text { if } \Phi=A_{n-1}, \\
\left(\begin{array}{l}
n \\
k
\end{array}\right)\left(\begin{array}{c}
m n \\
k
\end{array}\right), & \text { if } \Phi=B_{n} \text { or } C_{n}, \\
\left(\begin{array}{l}
n \\
k
\end{array}\right)\left(\begin{array}{c}
m n-m \\
k
\end{array}\right)+\left(\begin{array}{c}
n-2 \\
k-2
\end{array}\right)\left(\begin{array}{c}
m n-m+1 \\
k
\end{array}\right), & \text { if } \Phi=D_{n} .
\end{array}
$$


Remark 5.2. The number of $k$-element antichains in $\Phi^{+}$has been computed for the exceptional root systems by V. Reiner and J. McCammond, as shown in the following table.

TABLE 1 . The numbers $\mathrm{N}_{\Phi}(k)$ for the exceptional root systems.

\begin{tabular}{|c|l|l|l|l|l|l|l|l|l|}
\hline$k$ & 0 & 1 & 2 & 3 & 4 & 5 & 6 & 7 & 8 \\
\hline \hline$\Phi=G_{2}$ & 1 & 6 & 1 & & & & & & \\
\hline$\Phi=F_{4}$ & 1 & 24 & 55 & 24 & 1 & & & & \\
\hline$\Phi=E_{6}$ & 1 & 36 & 204 & 351 & 204 & 36 & 1 & & \\
\hline$\Phi=E_{7}$ & 1 & 63 & 546 & 1470 & 1470 & 546 & 63 & 1 & \\
\hline$\Phi=E_{8}$ & 1 & 120 & 1540 & 6120 & 9518 & 6120 & 1540 & 120 & 1 \\
\hline
\end{tabular}

In the remainder of this section we prove Proposition 5.1. We denote by $\mathrm{N}_{\Phi}^{m}(k)$ the number of $W$-orbits $x$ of $T^{m}$ with $r(x)=k$, which we simply write as $\mathrm{N}_{\Phi}(k)$ if $m=1$.

5.1. The case $\Phi=A_{n-1}$. The coroot lattice $\check{Q}$ is the lattice of points in $\mathbb{Z}^{n}$ with zero coordinate sum, $W$ is the symmetric group on $n$ elements which acts on $\check{Q}$ by permuting coordinates, and $h=n$. If $Q_{m, n}$ is the set of points in $\mathbb{Z}_{m n+1}^{n}$ having zero coordinate sum, on which $W$ also acts, then the natural map $\breve{Q} \rightarrow Q_{m, n}$ induces an isomorphism of $W$-modules $\check{Q} /(m n+1) \check{Q} \rightarrow Q_{m, n}$. Since $n$ is relatively prime to $m n+1$, it follows that the set $Q_{m, n}$ is in bijection with the set $\mathbb{Z}_{m n+1}^{n} /(1,1, \ldots, 1)$ of cosets of the cyclic subgroup of $\mathbb{Z}_{m n+1}^{n}$ generated by $(1,1, \ldots, 1)$, and this bijection commutes with the natural action of $W$ on the two sets. The stabilizer within $W$ of such a coset, represented say by $a=\left(a_{1}, a_{2}, \ldots, a_{n}\right) \in \mathbb{Z}_{m n+1}^{n}$, is generated by the transpositions in coordinates $i$ and $j$ for which $a_{i}=a_{j}$. It follows easily that $\mathrm{N}_{\Phi}^{m}(k)$ is equal to the number of $W$-orbits of such cosets for which $\left\{a_{1}, \ldots, a_{n}\right\}$ has $n-k$ elements, and hence to

$$
\frac{1}{m n+1}\left(\begin{array}{c}
n-1 \\
n-k-1
\end{array}\right)\left(\begin{array}{c}
m n+1 \\
n-k
\end{array}\right)
$$

which is equivalent to the proposed formula. It is known that another set of coset representatives for $\mathbb{Z}_{m n+1}^{n} /(1,1, \ldots, 1)$ is the set of $m$-parking functions of length $n$ 23], meaning integer sequences $a=\left(a_{1}, a_{2}, \ldots, a_{n}\right)$ such that the unique increasing rearrangement $\left(b_{1}, b_{2}, \ldots, b_{n}\right)$ of $a$ satisfies $0 \leq b_{i} \leq m(i-1)$ for all $i$. Thus $T^{m}$ is isomorphic as a $W$-module to the set of $m$-parking functions of length $n$, on which $W$ acts. The orbits of this action are indexed by the increasing parking functions, in other words the increasing integer sequences $\left(a_{1}, a_{2}, \ldots, a_{n}\right)$ with $0 \leq a_{i} \leq m(i-1)$ for each $i$. In the case $m=1$ it follows that $\mathrm{N}_{\Phi}(k)$ is equal to the number of lattice paths in $\mathbb{Z}^{2}$ with possible steps $(1,0)$ and $(0,1)$ which start at $(0,0)$, end at $(n, n)$, stay below the line $x=y$ and have $n-k-1$ northeast corners. The latter is a standard combinatorial interpretation of the Narayana number $\frac{1}{k+1}\left(\begin{array}{c}n \\ k\end{array}\right)\left(\begin{array}{c}n-1 \\ k\end{array}\right)$; see, for instance, [13, 24].

5.2. The case $\Phi=C_{n}$. We have $h=2 n, \check{Q}=\mathbb{Z}^{n}$, and $W$ is the hyperoctahedral group which acts on $\check{Q}$ by changing signs and permuting coordinates. If $[-m n, m n]=\{-m n, \ldots,-1,0,1, \ldots, m n\}$, then it is clear that $\check{Q} /(2 m n+1) \check{Q}$ is isomorphic as a $W$-module to $\mathbb{Z}_{2 m n+1}^{n}$, and hence to $[-m n, m n]^{n}$, on which $W$ 
acts. The orbits of this action are indexed by the increasing integer sequences $a=\left(a_{1}, a_{2}, \ldots, a_{n}\right)$ satisfying $0 \leq a_{i} \leq m n$. These sequences biject to lattice paths in $\mathbb{Z}^{2}$ which start at $(0,0)$ and end at $(n, m n)$, where the path corresponding to $a$ is the one which passes through the points $\left(i-1, a_{i}\right)$ and $\left(i, a_{i}\right)$ for $1 \leq i \leq n$. The stabilizer of $a$ within $W$ is minimally generated by the transpositions in coordinates $i$ and $i+1$ for those $i$ with $a_{i}=a_{i+1}$ and the sign change in the first coordinate, if $a_{1}=0$. It follows that $\mathrm{N}_{\Phi}^{m}(k)$ is equal to the number of increasing sequences $\left(a_{1}, a_{2}, \ldots, a_{n}\right)$ with $0 \leq a_{i} \leq m n$ such that the set $\left\{a_{1}, \ldots, a_{n}\right\}$ has $n-k$ nonzero elements, and hence to the number of lattice paths in $\mathbb{Z}^{2}$ which start at $(0,0)$, end at $(n, m n)$ and have $n-k$ northeast corners. The last statement implies that $\mathrm{N}_{\Phi}^{m}(k)=\left(\begin{array}{c}n \\ n-k\end{array}\right)\left(\begin{array}{c}m n \\ n-k\end{array}\right)$.

5.3. The case $\Phi=B_{n}$. We have $h=2 n, \check{Q}$ is the lattice of points in $\mathbb{Z}^{n}$ having even coordinate sum, and $W$ is as in the type $C_{n}$ case. By direct reasoning or by appealing to Lemma 4.1 we see that a set of representatives for the orbits of the $W$-action on $\check{Q} /(2 m n+1) \check{Q}$ is the set $O_{m, n}$ of (cosets of) increasing nonnegative integer sequences $a=\left(a_{1}, a_{2}, \ldots, a_{n}\right)$ such that $a_{1}+a_{2}+\cdots+a_{n}$ is even and

$$
a_{n-1}+a_{n} \leq 2 m n+1 \text {. }
$$

Moreover, the stabilizer of $a$ within $W$ is minimally generated by the transpositions in coordinates $i$ and $i+1$ for those $i$ with $a_{i}=a_{i+1}$, the sign change in the first coordinate, if $a_{1}=0$, and the element of $W$ which exchanges the last two coordinates and changes their signs, if $a_{n-1}+a_{n}=2 m n+1$. It follows that $\mathrm{N}_{B_{n}}^{m}(k)$ is equal to the number of sequences $a=\left(a_{1}, a_{2}, \ldots, a_{n}\right) \in O_{m, n}$ such that the set $\left\{a_{1}, \ldots, a_{n-1}, a_{n}, 2 m n+1-a_{n}\right\}$ has $n-k+1$ nonzero elements. Let $O_{m, n}^{\prime}$ be the set of increasing sequences $\left(a_{1}, a_{2}, \ldots, a_{n}\right)$ with $0 \leq a_{i} \leq m n$, considered in the type $C_{n}$ case. The map $f: O_{m, n}^{\prime} \rightarrow O_{m, n}$ which sends an element $\left(a_{1}, a_{2}, \ldots, a_{n}\right) \in O_{m, n}^{\prime}$ to

$$
\begin{cases}\left(a_{1}, \ldots, a_{n-1}, 2 a_{n}-a_{n-1}\right), & \text { if } a_{1}+\cdots+a_{n-2} \text { is even, } \\ \left(a_{1}, \ldots, a_{n-1}, 2 m n+1-2 a_{n}+a_{n-1}\right), & \text { if } a_{1}+\cdots+a_{n-2} \text { is odd, }\end{cases}
$$

is a bijection having the property that $\left\{b_{1}, \ldots, b_{n-1}, b_{n}, 2 m n+1-b_{n}\right\}$ has exactly one nonzero element more than $\left\{a_{1}, \ldots, a_{n}\right\}$, where $\left(b_{1}, \ldots, b_{n}\right)=f\left(a_{1}, \ldots, a_{n}\right)$; we leave it to the reader to check this. This shows that $\mathrm{N}_{B_{n}}^{m}(k)=\mathrm{N}_{C_{n}}^{m}(k)$.

5.4. The case $\Phi=D_{n}$. We have $h=2 n-2$, where $n \geq 3, \check{Q}$ is as in the type $B_{n}$ case, and $W$ is the index two subgroup of the hyperoctahedral group on $n$ elements which acts on $\check{Q}$ by changing an even number of signs and permuting coordinates. By direct reasoning or by appealing to Lemma 4.1, as in the previous case, we see that a set of representatives for the orbits of the $W$-action on $\check{Q} /(m h+1) \check{Q}$ is the set $O_{m, n}$ of (cosets of) integer sequences $a=\left(a_{1}, a_{2}, \ldots, a_{n}\right)$ such that $a_{1}+a_{2}+\cdots+a_{n}$ is even,

$$
0 \leq\left|a_{1}\right| \leq a_{2} \leq \cdots \leq a_{n}
$$

and

$$
a_{n-1}+a_{n} \leq 2 m(n-1)+1 .
$$

The stabilizer of $a$ within $W$ is minimally generated by the transpositions in coordinates $i$ and $i+1$ for those $i$ with $a_{i}=a_{i+1}$, the element of $W$ which exchanges 
the first two coordinates and switches their signs, if $a_{1}+a_{2}=0$, and that which exchanges the last two coordinates and switches their signs, if equality holds in (5.2). It follows that $\mathrm{N}_{\Phi}(k)$ is equal to the number of sequences $\left(a_{1}, a_{2}, \ldots, a_{n}\right) \in O_{m, n}$ such that the set $\left\{\left|a_{1}\right|, a_{2}, \ldots, a_{n-1}, a_{n}, 2 m(n-1)+1-a_{n}\right\}$ has $n-k$ nonzero elements, if $a_{1}=0$ and $a_{2}>0$, and $n-k+1$ nonzero elements otherwise. Let $O_{m, n}^{\prime}$ be the set of integer sequences $\left(a_{1}, a_{2}, \ldots, a_{n}\right)$ satisfying (5.1) and $a_{n} \leq m(n-1)$. As in the type $B_{n}$ case, the bijection $f: O_{m, n}^{\prime} \rightarrow O_{m, n}$ which sends an element $\left(a_{1}, a_{2}, \ldots, a_{n}\right) \in O_{m, n}^{\prime}$ to

$$
\left\{\begin{array}{lll}
\left(a_{1}, \ldots, a_{n-1}, 2 a_{n}-a_{n-1}\right), & \text { if } a_{1}+\cdots+a_{n-2} \text { is even, } \\
\left(a_{1}, \ldots, a_{n-1}, 2 m(n-1)+1-2 a_{n}+a_{n-1}\right), & \text { if } a_{1}+\cdots+a_{n-2} \text { is odd, }
\end{array}\right.
$$

shows that $\mathrm{N}_{\Phi}(k)$ is equal to the number of $\left(a_{1}, a_{2}, \ldots, a_{n}\right) \in O_{m, n}^{\prime}$ such that $\left\{\left|a_{1}\right|, a_{2}, \ldots, a_{n}\right\}$ has $n-k-1$ nonzero elements, if $a_{1}=0$ and $a_{2}>0$, and $n-k$ nonzero elements otherwise. The set of such sequences in $O_{m, n}^{\prime}$ with $a_{1}=a_{2}=0$ bijects to the set of lattice paths in $\mathbb{Z}^{2}$ starting at $(2,0)$, ending at $(n, m n-m)$ and having $n-k$ northeast corners, which has cardinality $\left(\begin{array}{c}n-2 \\ n-k\end{array}\right)\left(\begin{array}{c}m n-m \\ n-k\end{array}\right)$. Similar reasoning shows that there are $\left(\begin{array}{c}n-2 \\ n-k-2\end{array}\right)\left(\begin{array}{c}m n-m \\ n-k-1\end{array}\right)$ such sequences in $O_{m, n}^{\prime}$ with $a_{1}=0$ and $a_{2}>0$, and $2\left(\begin{array}{c}n-1 \\ n-k-1\end{array}\right)\left(\begin{array}{c}m n-m \\ n-k\end{array}\right)$ having $a_{1}$ nonzero. Hence

$$
\mathrm{N}_{\Phi}(k)=\left(\begin{array}{c}
n-2 \\
k-2
\end{array}\right)\left(\begin{array}{c}
m n-m \\
n-k
\end{array}\right)+\left(\begin{array}{c}
n-2 \\
k
\end{array}\right)\left(\begin{array}{c}
m n-m \\
n-k-1
\end{array}\right)+2\left(\begin{array}{c}
n-1 \\
k
\end{array}\right)\left(\begin{array}{c}
m n-m \\
n-k
\end{array}\right),
$$

which simplifies to the proposed formula.

\section{ACKNOWLEDGEMENTS}

I am grateful to Victor Reiner and Sergey Fomin for useful comments, and to an anonymous referee for pointing out a gap in the proof of one of the lemmas in Section 3 in an earlier version of the paper. I also thank Victor Reiner and Jon McCammond for their permission to present here the data shown in Table 1.

\section{REFERENCES}

1. C.A. Athanasiadis, Deformations of Coxeter hyperplane arrangements and their characteristic polynomials, in "Arrangements - Tokyo 1998" (M. Falk and H. Terao, eds.), Adv. Stud. Pure Math. 27, Kinokuniya, Tokyo, 2000, pp. 1-26. MR 2001i:52035

2. C.A. Athanasiadis, Generalized Catalan numbers, Weyl groups and arrangements of hyperplanes, Bull. London Math. Soc. (to appear).

3. D. Bessis, The dual braid monoid, Ann. Sci. Ecole Norm. Sup. 36 (2003), 647-683.

4. P. Cellini and P. Papi, ad-nilpotent ideals of a Borel subalgebra, J. Algebra 225 (2000), 130141. MR 2001g:17017

5. P. Cellini and P. Papi, ad-nilpotent ideals of a Borel subalgebra II, J. Algebra 258 (2002), $112-121$.

6. F. Chapoton, S. Fomin and A.V. Zelevinsky, Polytopal realizations of generalized associahedra, Canad. Math. Bull. 45 (2002), 537-566. MR 2003j:52014

7. D.Ž. Djoković, On conjugacy classes of elements of finite order in compact or complex semisimple Lie groups, Proc. Amer. Math. Soc. 80 (1980), 181-184. MR 81b:20052

8. S. Fomin and A.V. Zelevinsky, $Y$-systems and generalized associahedra, Ann. of Math. 158 (2003), 977-1018.

9. S. Fomin and A.V. Zelevinsky, Cluster algebras II: finite type classification, Invent. Math. 154 (2003), 63-121.

10. M.D. Haiman, Conjectures on the quotient ring by diagonal invariants, J. Algebraic Combin. 3 (1994), 17-76. MR 95a:20014 
11. J.E. Humphreys, Reflection groups and Coxeter groups, Cambridge Studies in Advanced Mathematics 29, Cambridge University Press, Cambridge, England, 1990. MR 92h:20002

12. T. Venkata Narayana, Sur les treillis formés par les partitions d'un entier et leurs applications à la théorie des probabilités, Comp. Rend. Acad. Sci. Paris 240 (1955), 1188-1189. MR 17:14a

13. T. Venkata Narayana, Lattice path combinatorics with statistical applications, University of Toronto Press, 1979. MR 81f:60019

14. D.I. Panyushev, Ad-nilpotent ideals of a Borel subalgebra: generators and duality, J. Algebra (to appear).

15. M. Picantin, Explicit presentations for the dual braid monoids, C. R. Math. Acad. Sci. Paris Sér. I Math. 334 (2002), 843-848. MR 2003d:20046

16. A. Postnikov and R.P. Stanley, Deformations of Coxeter hyperplane arrangements, J. Combin. Theory Ser. A 91 (2000), 544-597. MR 2003g:52032

17. V. Reiner, Non-crossing partitions for classical reflection groups, Discrete Math. 177 (1997), 195-222. MR 99f:06005

18. V. Reiner and V. Welker, On the Charney-Davis and Neggers-Stanley conjectures, preprint, 2002, 38 pages.

19. J.-Y. Shi, Alcoves corresponding to an affine Weyl group, J. London Math. Soc. 35 (1987), 42-55. MR 88g:20103a

20. J.-Y. Shi, Sign types corresponding to an affine Weyl group, J. London Math. Soc. 35 (1987), 56-74. MR 88g:20103b

21. J.-Y. Shi, The number of $\oplus$-sign types, Quart. J. Math. Oxford Ser. (2) 48 (1997), 93-105. MR 98c:20080

22. E. Sommers, B-stable ideals in the nilradical of a Borel subalgebra, ArXiV preprint math.RT/0303182, March 2003.

23. R.P. Stanley, Hyperplane arrangements, parking functions and tree inversions, in "Mathematical Essays in Honor of Gian-Carlo Rota" (B.E. Sagan and R.P. Stanley, eds.), Progress in Math. 161 (1998), Birkhäuser, Boston, pp. 359-375. MR 99f:05006

24. R.A. Sulanke, Catalan path statistics having the Narayana distribution, Discrete Math. 180 (1998), 369-389. MR 99b:05004

Department of Mathematics, University of Crete, 71409 Heraklion, Crete, Greece

E-mail address: caa@math.uoc.gr 